Homology, Homotopy and Applications, vol.15(2), 2013, pp.163-172

\title{
INVARIANTS PRESERVED BY MUTATION
}

THILO KUESSNER

\author{
(communicated by Charles A. Weibel)
}

\begin{abstract}
We prove that generalized mutation preserves several geometric invariants such as the volume and Goncharov invariant of closed or Q-rank 1 locally symmetric spaces.
\end{abstract}

\section{Introduction}

Rigidity results in the theory of locally symmetric spaces imply that geometrically defined invariants, such as the volume, are topological invariants. Yet it remains largely mysterious how these invariants are determined by the topology and how they behave with respect to topological operations such as cut and paste. "Cut and paste" hereby means the following operation: we have a properly embedded, 2 -sided, codimension 1 submanifold $\Sigma$ in a compact manifold $M$, and we denote by $M^{\tau}$ the manifold that is the result of cutting $M$ along $\Sigma$ and regluing via a diffeomorphism $\tau: \Sigma \rightarrow \Sigma$.

In [15] Ruberman considered the case of hyperbolic 3-manifolds and showed that mutation of a hyperbolic link yields a hyperbolic link of the same volume. More generally, he proved that for hyperbolic 3-manifolds $M$ and certain pairs $(\Sigma, \tau)$, especially for the hyperelliptic involution of the genus 2 surface, $M^{\tau}$ is always hyperbolic with $\operatorname{vol}\left(M^{\tau}\right)=\operatorname{vol}(M)$ if $\Sigma \subset M$ is incompressible and boundary-incompressible. (The latter conditions are needed only to guarantee hyperbolicity of $M^{\tau}$.) Neumann indicated in [14] that the $\operatorname{PSL}(2, \mathbf{C})$-fundamental class and the Bloch invariant of hyperbolic 3 -manifolds are also preserved under mutation. In $[\mathbf{1 0}]$ we gave a topological proof of Ruberman's theorem using the fundamental class construction. In [8] we used an analogous argument to prove that the volume of flag structures is also preserved under mutation. The aim of this paper is to prove in a general setting that $G$-fundamental classes and hence various geometric invariants are preserved under generalized mutation.

For a closed, orientable $d$-manifold $M$ and a representation $\rho: \pi_{1} M \rightarrow G$, one has the naturally associated $G$-fundamental class $(B \rho)_{*}[M] \in H_{d}(B G)$, where $B G$ denotes the classifying space with respect to the discrete topology on $G$. If $M$ has $\pi_{1}$-injective boundary, $G$ is a semisimple Lie group without compact factor, and $\rho$ sends $\pi_{1} \partial_{i} M$ to a parabolic subgroup $P_{i} \subset G$ for each component $\partial_{i} M$ of $\partial M$. Then we can still associate a fundamental class $(B \rho)_{*}[M, \partial M] \in H_{d}\left(B G^{\text {comp }}\right)$ for a certain completion $B G^{c o m p}$; see Section 4.1. We discuss in Section 3 that this

Received January 15, 2013; published on November 24, 2013. 2000 Mathematics Subject Classification: 57R19, 53C35, 57M50.

Key words and phrases: mutation, volume, Bloch invariant, locally symmetric space. Article available at http://intlpress.com/HHA/v15/n2/a10 and doi :10.4310/HHA.2013.v15.n2.a10 Copyright (C) 2013, International Press. Permission to copy for private use granted. 
fundamental class determines several geometric invariants. The generalized mutations to be considered will be defined in Definition 2.1. The general result proved in this paper is the following theorem:

Theorem 1.1. Assume $M$ is a compact, oriented d-manifold such that int $(M)$ is a Q-rank 1 locally symmetric space of noncompact type. Let $\rho: \pi_{1} M \rightarrow G$ be a representation to a semisimple Lie group without compact factor, sending the fundamental group of each boundary component $\pi_{1} \partial_{i} M$ to some parabolic subgroup $P_{i} \subset G$.

If $\rho^{\tau}: \pi_{1} M^{\tau} \rightarrow G$ is a generalized mutation of $\rho$, then

$$
(B \rho)_{*}[M, \partial M]_{\mathbf{Q}}=\left(B \rho^{\tau}\right)_{*}\left[M^{\tau}, \partial M^{\tau}\right]_{\mathbf{Q}} \in H_{d}\left(B G^{c o m p} ; \mathbf{Q}\right) .
$$

The corresponding result for $\mathbf{Z}$-coefficients is not true; examples are given in [14, Section 2.6]. We will see in Section 3 that the following invariants are determined by the rational $G$-fundamental class: $(B \rho)_{*}[M, \partial M]_{\mathbf{Q}}$. Thus their invariance under generalized mutations follows.

Corollary 1.2. The volume, the (generalized) rational Bloch invariants and Goncharov invariants of $\mathbf{Q}$-rank 1 locally symmetric spaces, the complex volume of representations and the rational Bloch invariant and volume of CR structures and flag structures are preserved under generalized mutations.

\section{Generalized mutation}

\subsection{Definition of generalized mutations}

In this paper we will consider the following situation: We have a compact manifold $M$ (possibly with boundary) and a properly embedded, 2-sided, codimension 1 submanifold $\Sigma \subset M$. We consider a diffeomorphism $\tau:(\Sigma, \partial \Sigma) \rightarrow(\Sigma, \partial \Sigma)$, and we denote $M^{\tau}$ the manifold, which is the result of cutting $M$ along $\Sigma$ and regluing via $\tau$.

We will also assume that a representation $\rho: \pi_{1} M \rightarrow G$ is given. This representation may arise from the identification of $\pi_{1} M$ with a discrete subgroup $\Gamma \subset G$ in case that $M=\Gamma \backslash G / K$ is a locally symmetric space of noncompact type, but we will also be interested in other representations, e.g., arising from CR or flag structures.

Definition 2.1 (Generalized mutation). For a fixed representation $\rho: \pi_{1} M \rightarrow G$, we say that a finite order homeomorphism $\tau: \Sigma \rightarrow \Sigma$ is a generalized mutation if there exists some finite order $A \in G$ with

$$
\rho\left(\tau_{*} h\right)=A \rho(h) A^{-1}
$$

for all $h \in \pi_{1} \Sigma$.

We remark that the condition of $A$ having finite order is implied if $\rho$ is the holonomy of a hyperbolic 3-manifold ([10, Observation 1.2]), or if $\rho\left(\pi_{1} \Sigma\right)$ is Zariski-dense.

Lemma 2.2. If $\tau: \Sigma \rightarrow \Sigma$ is a generalized mutation for a representation $\rho: \pi_{1} M$ $\rightarrow G$, then there is a representation

$$
\rho^{\tau}: \pi_{1} M^{\tau} \rightarrow G
$$

such that the restrictions of $\rho$ and $\rho^{\tau}$ to $\pi_{1}(M-\Sigma)$ agree. 
Proof. We can choose regular neighborhoods of $\Sigma$ in $M$ and $M^{\tau}$ and an identification of their complements. We denote $X$ the union of $M$ and $M^{\tau}$ along this identification. Let $\pi_{1} M=\langle S \mid R\rangle$ be a presentation of $\pi_{1} M$. The Seifert-van Kampen Theorem implies (with $i: \Sigma \rightarrow X$ denoting the inclusion):

$$
\pi_{1} X=\left\langle S, t \mid R, t i_{*}(h) t^{-1}=i_{*}\left(\tau_{*} h\right) \forall h \in \pi_{1} \Sigma\right\rangle .
$$

Definition 2.1 implies that we can extend $\rho$ to a representation $\rho^{X}: \pi_{1} X \rightarrow G$ by defining $\rho(t)=A$. Composition with $\pi_{1} M^{\tau} \rightarrow \pi_{1} X$ yields $\rho^{\tau}: \pi_{1} M^{\tau} \rightarrow G$.

\subsection{Examples}

Symmetries of representation variety

The mapping class of $\tau: \Sigma \rightarrow \Sigma$ acts on $R\left(\pi_{1} \Sigma, G\right)=\operatorname{Hom}\left(\pi_{1} \Sigma, G\right) / \sim$, the space of representations up to conjugation. The condition from Definition 2.1 is obviously equivalent to the condition that the conjugacy class of $\left.\rho\right|_{\pi_{1} \Sigma}$ is a fixed point for the action of $\tau_{*}$ on $R\left(\pi_{1} \Sigma, G\right)$. We do not know a general approach for finding such fixed points, but a remarkably general case is provided by the following example due to Ruberman. (Here $R_{d, f}\left(\pi_{1} \Sigma, G\right)$ is the subset of discrete, faithful, parabolicspreserving representations upon conjugation, an analogue of Teichmüller space.)

Example 2.3 (Surfaces in hyperbolic 3-manifolds). If $\Sigma \subset M$ is the genus 2 surface and $\tau: \Sigma \rightarrow \Sigma$ the hyperelliptic involution, then by [15, Theorem 2.2] $\tau_{*}$ acts trivially on $R_{d, f}\left(\pi_{1} \Sigma, S L(2, \mathbf{C})\right)$, every $\rho$ is a fixed point. The same is true for the $\tau$-invariant subsurfaces of $\Sigma$ : the 1- and 2-punctured torus and the 3- and 4-punctured sphere.

Example 2.4 (Totally geodesic submanifolds). If $M=\Gamma \backslash G / K$ is a closed locally symmetric space of noncompact type of dimension $d \geqslant 4, \Sigma \subset M$ a totally geodesic hypersurface and $\tau: \Sigma \rightarrow \Sigma$ a diffeomorphism, then by [5, Theorem IV.7.1] $\Sigma$ is a locally symmetric space, upon conjugation $\Sigma=H / K$ for some subgroup $H \subset G$. Hence Mostow rigidity means that $\tau: \Sigma \rightarrow \Sigma$ is homotopic to an isometry (of finite order), and there is some $A \in H \subset G$ with $\tau_{*}(h)=A h A^{-1}$ for all $h \in \pi_{1} \Sigma$.

\subsection{Discreteness}

Even though the invariants considered in this paper, including the volume and the Bloch invariant, are defined for arbitrary representations to a Lie group (not necessarily of discrete image), it is a natural question to ask whether the mutation $\rho^{\tau}$ of a discrete embedding $\rho: \pi_{1} M \rightarrow G$ is again discrete. In [15] Ruberman answered this question positively for lattices in $S L(2, \mathbf{C})$. When $\rho\left(\pi_{1} \Sigma\right)$ is geometrically finite (hence 1-quasifuchsian, [7, Definition 9.2]), then in [10, Proposition 3.1] we gave another proof by using the Maskit combination theorem from [13, Chapter VII]. The Maskit combination theorem in the formulation of $[\mathbf{1 3}]$ has an exact generalisation to higher-dimensional hyperbolic manifolds by the recent work of Li-Ohshika-Wang ([12, Theorem 4.2]). Thus one can literally adapt the proof of [10, Proposition 3.1] to obtain the following result:

Proposition 2.5. Let $M$ be a compact, oriented n-manifold such that $\operatorname{int}(M)$ is hyperbolic with holonomy $\rho: \pi_{1} M \rightarrow I_{\text {som }}{ }^{+}\left(H^{n}\right)$. Let $\Sigma \subset M$ be a properly embedded, 2 -sided, codimension 1 submanifold such that $\rho\left(\pi_{1} \Sigma\right)$ is geometrically finite, $(n-2)$ quasifuchsian. If $\tau: \Sigma \rightarrow \Sigma$ is a generalized mutation, then $\rho^{\tau}\left(\pi_{1} M^{\tau}\right)$ is a discrete subgroup of $\operatorname{Isom}^{+}\left(H^{n}\right)$. 


\subsection{The closed case}

Though this is superfluous from a logical point of view - as we handle the more general Q-rank 1 case in Section 4 - we first discuss the, technically simpler, proof of Theorem 1.1 for the closed case. The idea is essentially due to Neumann ([14], in the context of hyperbolic 3-manifolds), and in this case the simplicity and beauty of the argument can hopefully be better appreciated.

Let $X$ be constructed as in the proof of Lemma 2.2. The images of the fundamental classes of $M, M^{\tau}$ and the mapping torus $T^{\tau}$ satisfy the relation

$$
[M]-\left[M^{\tau}\right]=\left[T^{\tau}\right] \in H_{d}(X) .
$$

From the proof of Lemma 2.2, we have a representation $\rho^{X}: \pi_{1} X \rightarrow G$ with $\left.\rho^{X}\right|_{\pi_{1} M}=$ $\rho$ and $\left.\rho^{X}\right|_{\pi_{1} M^{\tau}}=\rho^{\tau}$. Then

$$
(B \rho)_{*}[M]-\left(B \rho^{\tau}\right)_{*}\left[M^{\tau}\right]=\left(B \rho^{X}\right)_{*}\left[T^{\tau}\right],
$$

and Theorem 1.1 for closed manifolds will follow once we have proved $\left(B \rho^{X}\right)_{*}\left[T^{\tau}\right]_{\mathbf{Q}}$ $=0$.

$\tau$ and $A$ have finite order, say $\tau^{n}=i d$ and $A^{n}=\mathbf{1}$ for an $n \in \mathbf{N}$. Hence we have an $n$-fold covering $p_{n}: \Sigma \times S^{1} \rightarrow T^{\tau}$. In the following diagram we denote by $i_{\Sigma}$ and $i_{T^{\tau}}$ the inclusions and by $P_{1}$ the projection to the first factor:

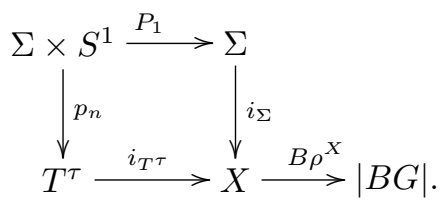

The left-hand square does not commute, even homologically, but we claim that the compositions with $B \rho^{X}$ commute up to homotopy and hence homologically.

Since $|B G|$ is aspherical ${ }^{1}$ it suffices to look at the fundamental group. We have $\pi_{1}\left(\Sigma \times S^{1}\right)=\pi_{1} \Sigma \oplus \mathbf{Z}$, and, for the $\pi_{1} \Sigma$-summand, of course already the left-hand square is commuting. Moreover, for the generator $s \in \pi_{1} S^{1}=\mathbf{Z}$ we have $\left(P_{1}\right)_{*}(s)=$ 0 . On the other hand, $\left(i_{T^{\tau}} p_{n}\right)_{*}(s)=t^{n}$, and thus $\left(B \rho_{X} i_{T^{\tau}} p_{n}\right)_{*}(s)=\rho_{X}\left(t^{n}\right)=A^{n}$ $=1$. Therefore, the induced homomorphisms of fundamental groups commute, and Hurewicz' Theorem² implies commutativity in homology.

With $d=\operatorname{dim}(M)$ we have $H_{d}(\Sigma)=0$. The homomorphism $\left(B \rho^{X} i_{\Sigma} P_{1}\right)_{d}$ factors over $H_{d}(\Sigma)$ and is therefore trivial. By the discussion before this implies

$$
\left(B \rho^{X} i_{T^{\tau}} p_{n}\right)_{d}\left[\Sigma \times \mathbf{S}^{1}\right]=0 .
$$

We have $\left(p_{n}\right)_{d}\left[\Sigma \times S^{1}\right]=n\left[T^{\tau}\right]$, thus rationally $\left(B \rho^{X} i_{T^{\tau}}\right)_{d}\left[T^{\tau}\right]_{\mathbf{Q}}=0$, hence the claim.

\footnotetext{
${ }^{1}$ In this paper, $|B G|$ always means the classifying space for $G$ with respect to the discrete topology. We think of it as the geometric realization of the simplicial set $B G$.

${ }^{2}$ Hurewicz' Theorem ([6, page 219]) gives $[X, Y]=\operatorname{Hom}\left(\pi_{1} X, \pi_{1} Y\right) / \operatorname{Inn}\left(\pi_{1} Y\right)$ for the homotopy classes of mappings from a space $X$ to an aspherical space $Y$. In particular, two maps are homotopic when they have the same effect on fundamental groups.
} 


\section{Invariants obtained from the $G$-fundamental class}

For a manifold $M$ and a group $G$, a representation $\rho: \pi_{1} M \rightarrow G$ induces a continuous map $B \rho: M \rightarrow|B G|$. If $M$ is a closed, oriented $d$-manifold with fundamental class $[M] \in H_{d}(M)$, then the $G$-fundamental class of $(M, \rho)$ is defined as $(B \rho)_{*}[M] \in H_{d}(B G)$.

More generally, we will be interested in the situation that $M$ has $\left(\pi_{1}\right.$-injective, possibly disconnected) boundary, $G$ is a semisimple Lie group without compact factors and the representation $\rho: \pi_{1} M \rightarrow G$ maps the fundamental group of each component $\partial_{i} M$ to some parabolic subgroup $P_{i} \subset G$. Then we have an element

$$
(B \rho)_{*}[M, \partial M] \in H_{d}\left(B G^{c o m p}\right) ;
$$

see Section 4.1 for its construction. Several invariants can be derived from this element.

\section{Volume of locally symmetric spaces}

If $\rho: \Gamma \rightarrow G$ is the inclusion of a Q-rank 1 lattice and $\operatorname{int}(M)=\Gamma \backslash G / K$ the locally symmetric space, then by $\left[\mathbf{9}\right.$, Section 4.2.3] there is the extended volume cocycle $\overline{c \nu}_{d}$ defined by

$$
\overline{c \nu}_{d}\left(g_{1}, \ldots, g_{d}\right)=\int_{\operatorname{str}\left(\tilde{x}, g_{1} \tilde{x}, \ldots, g_{1} \cdots g_{d} \tilde{x}\right)} d v o l
$$

for $\left(g_{1}, \ldots, g_{d}\right) \in B G$ and by

$$
\overline{c \nu}_{d}\left(p_{1}, \ldots, p_{d-1}, c_{i}\right)=\int_{\operatorname{str}\left(\tilde{x}, p_{1} \tilde{x}, \ldots, p_{1} \cdots p_{d-1} \tilde{x}, c_{i}\right)} d v o l
$$

for $\left(p_{1}, \ldots, p_{d-1}, c_{i}\right) \in$ Cone $(B G)$. Its cohomology class does not depend on $\tilde{x} \in G / K$ and by $[\mathbf{8}$, Lemma 6.3$]$ we have

$$
\left\langle\left[\overline{c \nu}_{d}\right],(B \rho)_{*}[M, \partial M]\right\rangle=\operatorname{vol}(M) .
$$

In particular, the volume is determined by $(B \rho)_{*}[M, \partial M]_{\mathbf{Q}}$.

\section{Goncharov invariant}

If again $\operatorname{int}(M)=\Gamma \backslash G / K$ is a Q-rank 1 locally symmetric space of finite volume, then by Weil rigidity we can assume that $\Gamma \subset G(\overline{\mathbf{Q}})$. If $\rho: \Gamma \rightarrow S L(N, \overline{\mathbf{Q}})$ is the restriction of some representation $G(\overline{\mathbf{Q}}) \rightarrow S L(N, \overline{\mathbf{Q}})$, then by [8, Proposition 7.1] the rational fundamental class

$$
(B \rho)_{*}[M, \partial M]_{\mathbf{Q}} \in H_{d}\left(B S L(N, \overline{\mathbf{Q}})^{c o m p}, \mathbf{Q}\right)
$$

has a (unique) preimage

$$
\bar{\gamma}(M) \in H_{d}(B S L(N, \overline{\mathbf{Q}}), \mathbf{Q})
$$

and also an associated element

$$
\gamma(M) \in K_{d}(\overline{\mathbf{Q}}) \otimes \mathbf{Q},
$$

which are called the homological and $K$-theoretic Goncharov invariant, respectively. Similarly, if $\mathbf{F} \subset \mathbf{C}$ is a subring with 1 and $\rho(\Gamma) \subset S L(N, \mathbf{F})$, then one obtains $\gamma(M) \in$ $K_{d}(\mathbf{F}) \otimes \mathbf{Q}$. By definition, $\gamma(M)$ is determined by $(B \rho)_{*}[M, \partial M]_{\mathbf{Q}}$. 


\section{Complex volume}

If $M$ is a closed 3-manifold and $\rho: \pi_{1} M \rightarrow S L(n, \mathbf{C})$ a representation, then the complex volume as defined in $[4]$ is

$$
\frac{1}{i}\left\langle\hat{c},(B \rho)_{*}[M]\right\rangle
$$

where $\hat{c}$ is the Cheeger-Chern-Simons class of the flat bundle with monodromy $\rho$.

\section{Bloch invariant}

It is proved in the appendix of [2] that the Bloch-Wigner morphism sends the $P S L(2, \mathbf{C})$-fundamental class of a closed hyperbolic 3-manifold to its Bloch invariant $\beta(M)$. The corresponding result for cusped hyperbolic 3-manifolds, as well as for the generalized Bloch invariant of (closed or R-rank 1) locally symmetric spaces, has been proved in [11, Theorem 1]; see also [8, Definition 8.1] for Q-rank 1 spaces. So the rational Bloch-Wigner morphism maps $(B \rho)_{*}[M, \partial M]_{\mathbf{Q}}$ to the rational Bloch invariant $\beta(M) \otimes 1$.

\section{Bloch invariant of CR structures ([3])}

If $M$ is a finite-volume hyperbolic manifold and

$$
\rho: \pi_{1} M \rightarrow S U(2,1)
$$

a reductive, boundary-unipotent representation, then [8, Lemma 10.3] implies that the Bloch invariant $\beta_{F W}(M)$ is determined by $(B \rho)_{*}[M, \partial M]$.

Bloch invariant and volume of flag structures ([1])

If $M$ is a finite-volume hyperbolic 3 -manifold, and

$$
h: \mathbf{C} P^{1} \rightarrow \mathcal{F l}\left(\mathbf{C}^{3}\right)
$$

is equivariant with respect to some representation

$$
\rho: \pi_{1} M \rightarrow S L(3, \mathbf{C}),
$$

then Bergeron-Falbel-Guilloux define a Bloch invariant $\beta_{h}(M)$, which generalizes the Bloch invariants of hyperbolic and CR structures. It follows from [8, Lemma 10.7] that $\beta_{h}(M)$ is determined by $(B \rho)_{*}[M, \partial M]$. In particular, the volume of the flag structure is determined by $(B \rho)_{*}[M, \partial M]_{\mathbf{Q}}$.

\section{Proof}

\subsection{Recollections from [9, Section 4]: Construction of $(B \rho)_{*}[M, \partial M]$}

Definition 4.1. For a manifold $M$ and $\partial_{1} M, \ldots, \partial_{s} M$ the components of $\partial M$, we let

$$
\text { DCone }\left(\cup_{i=1}^{s} \partial_{i} M \rightarrow M\right)
$$

be the union along $\partial M$ of $M$ with the (disjoint) cones over $\partial_{1} M, \ldots, \partial_{s} M$.

For a group $\Gamma$ and a collection of subgroups $\Gamma_{1}, \ldots, \Gamma_{s}$, we denote by

$$
B \Gamma^{\text {comp }}:=\text { DCone }\left(\cup_{i=1}^{s} B \Gamma_{i} \rightarrow B \Gamma\right)
$$

the union along $\cup_{i=1}^{s} B \Gamma_{i}$ of the simplicial set $B \Gamma$ with the cones over $B \Gamma_{i}$. 
If $G$ is a semisimple Lie group without compact factor and $\partial_{\infty} G / K$ the Hadamard boundary of the associated symmetric space, then we denote ${ }^{3}$

$$
B G^{\text {comp }}=\text { DCone }\left(\dot{\cup}_{c \in \partial_{\infty} G / K} B P_{c} \rightarrow B G\right),
$$

where $P_{c}$ is the subgroup of parabolic elements fixing $c$.

Let $M$ be connected and the components $\partial_{1} M, \ldots, \partial_{s} M$ be $\pi_{1}$-injective. We fix $x \in M, x_{i} \in \partial_{i} M$ and pathes $l_{i}:[0,1] \rightarrow M$ with $l_{i}(0)=x, l_{i}(1)=x_{i}$ for $1 \leqslant i \leqslant s$. Conjugation by $l_{i}$ identifies $\pi_{1}\left(\partial_{i} M, x_{i}\right)$ to a subgroup $\Gamma_{i} \subset \Gamma:=\pi_{1}(M, x)$.

Let $|B \Gamma|$ denote the geometric realization of the simplicial set $B \Gamma$. The classifying map $\Psi_{M}: M \rightarrow|B \Gamma|$ extends to $\Psi_{M}:$ DCone $\left(\cup_{i=1}^{s} \partial_{i} M \rightarrow M\right) \rightarrow\left|B \Gamma^{\text {comp }}\right|$.

For $* \geqslant 2$ we have an isomorphism $H_{*}(M, \partial M) \cong H_{*}\left(D C o n e\left(\cup_{i=1}^{s} \partial_{i} M \rightarrow M\right)\right)$; see [8, Lemma 5.1]. Thus, if $\operatorname{dim}(M) \geqslant 2$, then $\left(\Psi_{M}\right)_{*}[M, \partial M] \in H_{*}\left(\left|B \Gamma^{\text {comp }}\right|\right)$ is defined.

We assume that $\rho: \Gamma \rightarrow G$ is a representation to a simple Lie group without compact factor, sending each $\Gamma_{i}$ to a parabolic subgroup $P_{i} \subset$ Fix $\left(c_{i}\right), c_{i} \in \partial_{\infty} G / K$, where $G / K$ is the associated symmetric space. (This holds, in particular, for the inclusion $\rho: \Gamma \rightarrow G$ if $\operatorname{int}(M) \cong \Gamma \backslash G / K$ is a Q-rank 1 locally symmetric space.)

Then we have a well-defined simplicial map $B \rho: B \Gamma^{\text {comp }} \rightarrow B G^{\text {comp }}$ by sending the cone point over $\Gamma_{i}$ to $c_{i}$. It induces the continuous map $|B \rho|:\left|B \Gamma^{\text {comp }}\right| \rightarrow\left|B G^{\text {comp }}\right|$. We will use the shorthand

$$
(B \rho)_{*}[M, \partial M] \in H_{*}^{s i m p}\left(B G^{c o m p}\right)
$$

for the image of $\left(|B \rho| \Psi_{M}\right)_{*}[M, \partial M] \in H_{*}\left(\left|B G^{\text {comp }}\right|\right)$ under the canonical isomorphism $H_{*}\left(\left|B G^{\text {comp }}\right|\right) \cong H_{*}^{\text {simp }}\left(B G^{\text {comp }}\right)$.

\subsection{Proof of Theorem 1.1}

Proof. The proof is similar to that of [8, Corollary 10.8] and [10, Theorem 1]. Let $X$ be constructed as in the proof of Lemma 2.2 and let $\partial X=X \cap\left(\partial M \cup \partial M^{\tau}\right)$. By Lemma 2.2 we have a representation $\rho^{X}: \pi_{1} X \rightarrow G$ with $\left.\rho^{X}\right|_{\pi_{1} M}=\rho$ and $\rho^{X}(t)=A$. The construction of $X$ implies that

$$
i_{M *}[M, \partial M]-i_{M^{\tau} *}\left[M^{\tau}, \partial M^{\tau}\right]=i_{T^{\tau} *}\left[T^{\tau}, \partial T^{\tau}\right] \in H_{d}(X, \partial X),
$$

where $i_{M}, i_{M^{\tau}}, i_{T^{\tau}}$ are the inclusions of $M, M^{\tau}$ and the mapping torus $T^{\tau}$ into $X$.

Fix $n \in \mathbf{N}$ such that $A^{n}=\mathbf{1}$ and $\tau^{n}=i d$. Using the presentation

$$
\pi_{1} X=\left\langle S, t \mid R, t\left(i_{\Sigma *} h\right) t^{-1}=i_{\Sigma_{*}}\left(\tau_{*} h\right) \forall h \in \pi_{1} \Sigma\right\rangle,
$$

we define a surjective homomorphism $a: \pi_{1} X \rightarrow \mathbf{Z} / n \mathbf{Z}$ by

$$
a(t)=1, a(s)=0 \forall s \in S .
$$

Let $\pi: \widehat{X} \rightarrow X$ be the $n$-fold cyclic covering with $\Gamma^{\widehat{X}}:=\pi_{1}(\widehat{X}, \hat{x}) \cong \operatorname{ker}(a)$. Consider $\widehat{M}=\pi^{-1}(M), \widehat{M}^{\tau}=\pi^{-1}\left(M^{\tau}\right)$ and $\Sigma \times \mathbf{S}^{1}=\pi^{-1}\left(T^{\tau}\right)$.

\footnotetext{
${ }^{3}$ In $[\mathbf{9}]$ we were using the larger space DCone $\left(\dot{\cup}_{c \in \partial_{\infty} G / K} B G \rightarrow B G\right)$. Even though this does not
} affect any of the arguments, the 'smaller' definition of $B G^{c o m p}$ might seem a more natural one. 
The transfer map $\operatorname{tr}: H_{*}(X, \partial X) \rightarrow H_{*}(\widehat{X}, \partial \widehat{X})$ applied to Equation (1) yields

$$
i_{\widehat{M} *}[\widehat{M}, \partial \widehat{M}]-i_{\widehat{M}^{\tau} *}\left[\widehat{M}^{\tau}, \partial \widehat{M}^{\tau}\right]=i_{\Sigma \times \mathbf{S}^{1} *}\left[\Sigma \times \mathbf{S}^{1}, \partial \Sigma \times \mathbf{S}^{1}\right] \in H_{d}(\widehat{X}, \partial \widehat{X}) .
$$

Let $\partial_{1} M, \ldots, \partial_{s} M$ be the components of $\partial M$. The path $l_{i}$, which defines the isomorphism of $\pi_{1}\left(\partial_{i} M, x_{i}\right)$ with $\Gamma_{i} \subset \Gamma:=\pi_{1}(M, x)$ (see Section 3.1), also identifies $\pi_{1}\left(\partial_{i} X\right)$ to a subgroup $\Gamma_{i}^{X} \subset \pi_{1}(X, x)$ for the corresponding component $\partial_{i} X$ of $\partial X$. For each component $\partial_{i k} \widehat{X}$ of $\pi^{-1}\left(\partial_{i} X\right)$, we can use a lift $\hat{l}_{i k}$ of $l_{i}$ to define an isomorphism from $\pi_{1}\left(\partial_{i k} \widehat{X}, \hat{x}_{i k}\right)$ to a subgroup $\Gamma_{i k}^{\widehat{X}} \subset \Gamma^{\widehat{X}}$ with $\pi_{*}\left(\Gamma_{i k}^{\widehat{X}}\right)=\Gamma_{i}^{X}$.

The representation $\rho_{\widehat{X}}:=\rho^{X} \pi_{*}: \Gamma^{\widehat{X}} \rightarrow G$ sends all $\Gamma_{i k}^{\widehat{X}}$ to the parabolic group $P_{i}$. Indeed, $\Gamma_{i}^{X}=\pi_{*}\left(\Gamma_{i k}^{\widehat{X}}\right)$ is generated by $t^{n}$ and elements of $\Gamma_{i}$, and since $\rho_{X}\left(t^{n}\right)=$ $A^{n}=\mathbf{1}$ we have $\rho_{\widehat{X}}\left(\Gamma_{i}^{\widehat{X}}\right)=\rho_{X}\left(\Gamma_{i}^{X}\right)=\rho_{X}\left(\Gamma_{i}\right)=\rho\left(\Gamma_{i}\right) \subset P_{i}$. Therefore, we can extend $B \rho_{\widehat{X}}$ to

$$
B \rho_{\widehat{X}}:\left(B \Gamma^{\widehat{X}}\right)^{c o m p} \rightarrow B G^{c o m p}
$$

by mapping the cone point over $B \Gamma_{i k}^{\widehat{X}}$ to $c_{i}$, the fixed point of the parabolic group $P_{i}$.

Let $\Psi_{\widehat{X}}:$ DCone $\left(\cup_{i, k} \partial_{i k} \widehat{X} \rightarrow \widehat{X}\right) \rightarrow\left|B \Gamma^{\widehat{X} c o m p}\right|$ be the extension of the classifying map. We look at the effect of $\left|B \rho_{\widehat{X}}\right| \Psi_{\widehat{X}} i_{\Sigma \times \mathbf{S}^{1}}$ on $\pi_{1}\left(\Sigma \times \mathbf{S}^{1}\right)$ (and on its subgroups isomorphic to images of $\pi_{1}\left(\partial_{j} \Sigma \times \mathbf{S}^{1}\right)$ for the components $\partial_{j} \Sigma$ of $\left.\partial \Sigma\right)$. The generator $z$ of $\pi_{1} \mathbf{S}^{1}$ is sent to $\rho_{\widehat{X}}(z)=\rho_{X}\left(t^{n}\right)=A^{n}=\mathbf{1}$ and elements of $\pi_{1} \Sigma$ are sent to their image under $\rho_{\widehat{X}}$. Thus the effect on the fundamental group of $\Sigma \times \mathbf{S}^{1}$ (and its subgroups) is the same as for $\left|B \rho_{\widehat{X}}\right| \Psi_{\widehat{X}} i_{\Sigma} p_{1}$, where $p_{1}:\left(\Sigma \times \mathbf{S}^{1}, \Sigma \times \mathbf{S}^{1}\right) \rightarrow(\Sigma, \partial \Sigma)$ means projection to the first factor.

By asphericity of $|B G|$ and Hurewicz' Theorem ([6, page 219]), this implies that $\left|B \rho_{\widehat{X}}\right| \Psi_{\widehat{X}} i_{\Sigma \times \mathbf{S}^{1}}$ and $\left|B \rho_{\widehat{X}}\right| \Psi_{\widehat{X}} i_{\Sigma} p_{1}$ are homotopic as maps from $\Sigma \times \mathbf{S}^{1}$ to $|B G|$. The same argument applied to the path-components $\partial_{j} \Sigma \times \mathbf{S}^{1}$ of $\partial \Sigma \times \mathbf{S}^{1}$ and to the aspherical space $\left|B P_{i}\right|$ (with $P_{i}$ the parabolic group corresponding to the component $\partial_{i} M \supset \partial_{j} \Sigma$ ) implies that the restrictions of both maps are homotopic as mappings from $\partial_{j} \Sigma \times \mathbf{S}^{1}$ to $\left|B P_{i}\right|$. The cone of the homotopy is then a homotopy between the corresponding maps from $\operatorname{Cone}\left(\partial_{j} \Sigma \times \mathbf{S}^{1}\right)$ to Cone $\left(\left|B P_{i}\right|\right)$.

Now, $\left|B G^{\text {comp }}\right|$ is a homotopy colimit of the cofibrant diagram

$$
\cup_{i} \text { Cone }\left(\left|B P_{i}\right|\right) \leftarrow \cup_{i}\left|B P_{i}\right| \rightarrow|B G|,
$$

and DCone $\left(\cup_{j} \partial_{j} \Sigma \times \mathbf{S}^{1} \rightarrow \Sigma \times \mathbf{S}^{1}\right)$ is a homotopy colimit of the cofibrant diagram

$$
\cup_{j} \text { Cone }\left(\partial_{j} \Sigma \times \mathbf{S}^{1}\right) \leftarrow \cup_{j} \partial_{j} \Sigma \times \mathbf{S}^{1} \rightarrow \Sigma \times \mathbf{S}^{1} .
$$

So homotopy invariance of homotopy colimits $([\mathbf{1 6}$, Section 5$])$ gives a homotopy

$$
\left|B \rho_{\widehat{X}}\right| \Psi_{\widehat{X}} i_{\Sigma \times \mathbf{S}^{1}} \sim\left|B \rho_{\widehat{X}}\right| \Psi_{\widehat{X}} i_{\Sigma} p_{1}: \text { DCone }\left(\cup_{j} \partial_{j} \Sigma \times \mathbf{S}^{1} \rightarrow \Sigma \times \mathbf{S}^{1}\right) \rightarrow\left|B G^{c o m p}\right| .
$$

We use [8, Lemma 5.2] to identify $H_{*}(\Sigma, \partial \Sigma)$ with $H_{*}\left(D C o n e\left(\cup_{j} \partial_{j} \Sigma \rightarrow \Sigma\right)\right)$ in degrees $* \geqslant 2$ and obtain

$$
\left(\left|B \rho_{\widehat{X}}\right| \Psi_{\widehat{X}} i_{\Sigma \times \mathbf{S}^{1}}\right)_{*}=\left(\left|B \rho_{\widehat{X}}\right| \Psi_{\widehat{X}} i_{\Sigma} P_{1}\right)_{*}: H_{d}\left(\Sigma \times \mathbf{S}^{1}, \partial \Sigma \times \mathbf{S}^{1}\right) \rightarrow H_{d}\left(\left|B G^{c o m p}\right|\right) .
$$


Since the latter homomorphism factors over $H_{d}(\Sigma, \partial \Sigma)=0$, we have

$$
\left(\left|B \rho_{\widehat{X}}\right| \Psi_{\widehat{X}} i_{\Sigma \times \mathbf{S}^{1}}\right)_{*}\left[\Sigma \times \mathbf{S}^{1}, \partial \Sigma \times \mathbf{S}^{1}\right]=0,
$$

and Equation (2) implies

$$
\begin{aligned}
\left(\left|B \rho_{\widehat{X}}\right| \Psi_{\widehat{X}} i_{\widehat{M}}\right)_{*}[\widehat{M}, \partial \widehat{M}] & =\left(\left(\left|B \rho_{\widehat{X}}\right| \Psi_{\widehat{X}} i_{\widehat{M}^{\tau}}\right)_{*}\left[\widehat{M}^{\tau}, \partial \widehat{M}^{\tau}\right] \in H_{d}\left(\left|B G^{c o m p}\right|\right) .\right.
\end{aligned}
$$

On the other hand we have

$$
\begin{aligned}
(B \rho)_{*}[M, \partial M]_{\mathbf{Q}} & :=\left(|B \rho| \Psi_{M}\right)_{*}[M, \partial M]_{\mathbf{Q}} \\
& =\frac{1}{n}\left(\left|B \rho_{X}\right| \Psi_{X} i_{M}\right)_{*} \pi_{*}[\widehat{M}, \partial \widehat{M}]_{\mathbf{Q}}=\frac{1}{n}\left(\left|B \rho_{\widehat{X}}\right| \Psi_{\widehat{X}} i_{\widehat{M}}\right)_{*}[\widehat{M}, \partial \widehat{M}]_{\mathbf{Q}},
\end{aligned}
$$

similarly for $M^{\tau}$, and thus Equation (3) implies the claim of Theorem 1.

\section{References}

[1] N. Bergeron, E. Falbel and A. Guilloux, Tetrahedra of flags, volume and homology of SL(3), preprint, http://arxiv.org/abs/1101.2742.

[2] J.L. Dupont and C.H. Sah, Scissors congruences. II, J. Pure Appl. Algebra 25 (1982), no. 2, 159-195.

[3] E. Falbel and Q. Wang, A combinatorial invariant for spherical CR structures, Asian J. Math., to appear, http://arxiv.org/abs/1007.5228.

[4] S. Garoufalidis, D. Thurston and C. Zickert, Complex volume of SL(n,C)representations of 3-manifolds, preprint, http://arxiv.org/abs/1111.2828.

[5] S. Helgason, Differential geometry and symmetric spaces, Academic Press, New York (1962).

[6] W. Hurewicz, Beiträge zur Theorie der Deformationen IV: Asphärische Räume, Proc. Akad. Wetensch. Amsterdam 39 (1936), 215-224.

[7] M. Kapovich, Kleinian groups in higher dimensions, in Geometry and dynamics of groups and spaces, Progr. Math. 265 (2008), 487-564, Birkhäuser, Basel.

[8] I. Kim, S. Kim and T. Kuessner, Homological and Bloch invariants for $\mathbb{Q}$-rank one spaces and flag structures, preprint, http://arxiv.org/abs/1210.7058.

[9] T. Kuessner, Locally symmetric spaces and $K$-theory of number fields, Algebr. Geom. Topol. 12 (2012), no. 1, 155-213.

[10] T. Kuessner, Mutation and recombination for hyperbolic 3-manifolds, $J$. Gökova Geom. Topol. GGT 5 (2011), 20-30.

[11] T. Kuessner, Group homology and ideal fundamental cycles, Topology Proc. 40 (2012), 239-258.

[12] L. Li, K. Ohshika and X. Wang, On Klein-Maskit combination theorem in space. I, Osaka J. Math. 46 (2009), no. 4, 1097-1141.

[13] B. Maskit, Kleinian groups, Grundlehren der Mathematischen Wissenschaften 287, Springer-Verlag, New York (1988). 
[14] W. Neumann, Realizing arithmetic invariants of hyperbolic 3-manifolds, in Interactions between hyperbolic geometry, quantum topology and number theory, Contemp. Math. 541, 233-246, AMS, Providence, RI (2011).

[15] D. Ruberman, Mutation and volumes of knots in $S^{3}$, Invent. Math. 90 (1987), no. 1, 189-215.

[16] R.M. Vogt, Homotopy limits and colimits, Math. Z. 134 (1973), no. 1, 11-52.

Thilo Kuessner kuessner@kias.re.kr

School of Mathematics, Korea Institute for Advanced Study, 85 Hoegiro, Dongdaemun-gu, Seoul 130-722, Republic of Korea 